\title{
Female preference for dynamic traits in the green swordtail, Xiphophorus helleri
}

\author{
GIL G. ROSENTHAL, CHRISTOPHER S. EVANS \& WILLIAM L. MILLER \\ A nimal Communication L aboratory, U niversity of California at Davis, U.S.A. \\ ( Received 25 J anuary 1995; initial acceptance 8 M ay 1995; \\ final acceptance 31 J uly 1995; M S. number: A7242)
}

\begin{abstract}
A bstract. A nalyses of the relationship between female preference and male behaviour have been complicated by correlated variation in factors such as male size and appearance. This study examined the effects of systematically manipulating male behaviour, while holding male morphology constant. $\mathrm{F}$ emale green swordtails were shown video-recorded sequences of the same male engaging in an active courtship display, performing similar levels of feeding activity, and remaining inactive. Control sequences of moving food particles and of an empty aquarium were also presented. F emale responsiveness was significantly different across time intervals (before, during and after the stimulus) for the three stimuli showing a male, but not for the two controls. A nalyses of female behaviour patterns during the stimulus presentations revealed that they preferred sequences of courting males to all other stimuli. F emales did not respond differently to the feeding and inactive sequences. These results indicate that female interest depends upon a specific set of motor patterns, and suggest that male behaviour and morphology may act synergistically to determine female preference. Video stimuli should prove useful in future experiments seeking to identify the role of specific courtship motor patterns in mate choice.

(C) 1996 The A ssociation for the Study of A nimal Behaviour
\end{abstract}

R ecent models of sexual selection have considered the role of perceptual mechanisms in the evolution of sexually dimorphic traits. Ryan (1990) proposed that secondary sexual characteristics can evolve to exploit pre-existing sensory biases within individuals of the opposite sex, and Endler (1992) examined the evolution of male morphology in terms of constraints imposed by environmental parameters and predator pressures. Support for these models has been found in a variety of systems involving both visual signals (Basolo 1990a; Proctor 1991; Endler 1992) and acoustic signals ( $R$ yan 1991).

$M$ ost studies of the interaction between sexual signals and sensory systems, like most studies of sexual selection in general, have concentrated on single traits. Considerable evidence suggests, however, that mate choice decisions are typically

Correspondence: G. G. Rosenthal, Department of Zoology, U niversity of Texas, A ustin, TX 78712, U.S.A. (email: FISH M AN@M AIL.UTEXAS.EDU).C. S. Evans is at the D epartment of Psychology, School of Behavioural Sciences, M acquarie U niversity, Sydney, N SW 2109, A ustralia. W. L. M iller is at the Center for N euroscience, U niversity of California, Davis, CA 95616, U.S.A. dependent upon the integration of information from a number of characters. Burley (1981) showed that preference by female pigeons, Columba livia, could be expressed as a function of male coloration, rank order and prior sexual experience. Female red jungle fowl, Gallus gallus, attend to several male traits, including tail length, hackle feather colour, comb length and colour, and courtship display (Zuk et al. 1992). Similarly, female great tits, $P$ arus major, select males on the basis of both song repertoire size and stripe size (Baker et al. 1986). Female guppies, Poecilia reticulata, attend to tail size (Bischoff et al. 1985), display rate ( $F$ arr 1980), colour contrast (Endler 1980), brightness (K odric-Brown 1985), and orange coloration (Houde 1987). Evaluation of multiple male ornaments may yield a more accurate estimate of a male's current physical condition, as well as provide a degree of redundancy that reduces the likelihood of erroneous rejection based on one character alone (Zuk et al. 1992).

Studies of the auditory basis of female mate choice suggest that it is useful to categorize traits as either 'static' or 'dynamic', depending on the level of within-male variability during a calling 
bout (Gerhardt 1991). In treefrogs (family Hylidae), static properties are relatively stable (mean coefficient of variation $<4 \%$ ), while dynamic properties are much more labile (mean coefficient of variation $>12 \%$ ). Playback experiments, in which tape-recorded vocalizations or computer-generated sounds are substituted for conspecific males, have allowed systematic manipulations of signal morphology so that the parameters involved in mate choice by females can be identified with confidence (reviewed by Gerhardt 1988). These studies reveal that female responsiveness is dependent upon information from both static and dynamic characters of male advertisement calls (G erhardt 1991).

In contrast, experimental analysis of visual signals has been largely confined to the manipulation of static traits, such as the size and shape of male ornaments (A ndersson 1982; Basolo 1990a; Hill 1990; Zuk et al. 1992). Observational studies of natural interactions suggest that dynamic male displays may also be important (Farr 1980; A ndersson 1982; Ryan \& Wagner 1987; Clayton 1990), because there are reliable correlations between male display quality and female preference. These findings raise the possibility that dynamic traits may be a major factor in sexual selection. Very few studies, however, have explored the effects of manipulating dynamic traits because of a serious technical impediment: the absence of a visual analogue to the auditory playback experiment.

It is difficult, if not impossible, to control courtship behaviour when using live males as stimuli, and artificial models are poorly suited for simulating complex displays (review in Evans $\& M$ arler 1991). Video-recordings can, however, readily be edited to produce a specific array of stimuli with appropriate variation in either behaviour or morphology. Sequences can be created that show only selected motor patterns, and a standard stimulus can be presented to all subjects, avoiding the confounding effects of differences in male behaviour and of interactions between the male and female (e.g. Collins 1994). This approach thus provides all of the benefits of the playback techniques that have proved so successful in analyses of acoustic signals over the last four decades (reviewed by M cG regor 1992).

R ecent experiments have used presentations of video image sequences to identify the visual cues mediating species recognition, mate choice and predator recognition. Clark \& U etz $(1990,1992)$ used both analogue and digital video stimuli to present female jumping spiders, $M$ aevia inclemens, with the courtship displays of two male morphs. This approach allowed them to manipulate male morphology and behaviour independently and to quantify the way in which these factors interact to affect female responsiveness. Video stimuli have also been successfully used to examine antipredator responses in chickens ( $E$ vans $\& M$ arler 1991, 1992; Evans et al. 1993a, b) and recognition of conspecific intruders by A nolis lizards (M acedonia et al. 1994). In the present study, we used a similar video playback technique to assess the role of dynamic traits in mate choice by female green swordtails, Xiphophorus helleri (Pisces: Poeciliidae).

The green swordtail has received attention since D arwin (1871) cited it as a striking illustration of sexual dimorphism and male ornament. Male $X$. helleri have the lower rays of the caudal fin modified into a long 'sword', which in some cases approaches or exceeds the male's own standard length. Experiments by Basolo (1990b), in which sword length was surgically manipulated, demonstrated that females prefer males with longer swords. The sword is, however, part of a larger visual context that includes the size and appearance of the entire male, together with an extensive set of motor patterns specific to courtship (Schlosberg et al. 1949; Clark et al. 1954; F ranck 1964; Hemens 1966). Observational studies of several other poeciliids suggest that female choice is also influenced by male courtship display. F arr (1980) found a positive correlation between mating success and the rate at which male guppies courted. In X. nigrensis, females preferred larger males performing a sexual display to non-courting, smaller males (Ryan et al. 1990). $X$ iphophorus pygmaeus females even preferred larger courting $X$. nigrensis to their non-courting conspecifics (R yan \& Wagner 1987).

The goal of the present study was to examine the effects of variation in male behaviour on female responsiveness. Females were presented with video playbacks of courting males and of the same male engaged in a similar level of noncourtship (feeding) activity, or inactive. Control sequences presented images of moving food particles and of an empty aquarium. These stimuli allowed us to assess systematically the effects of dynamic male traits, while holding constant those 


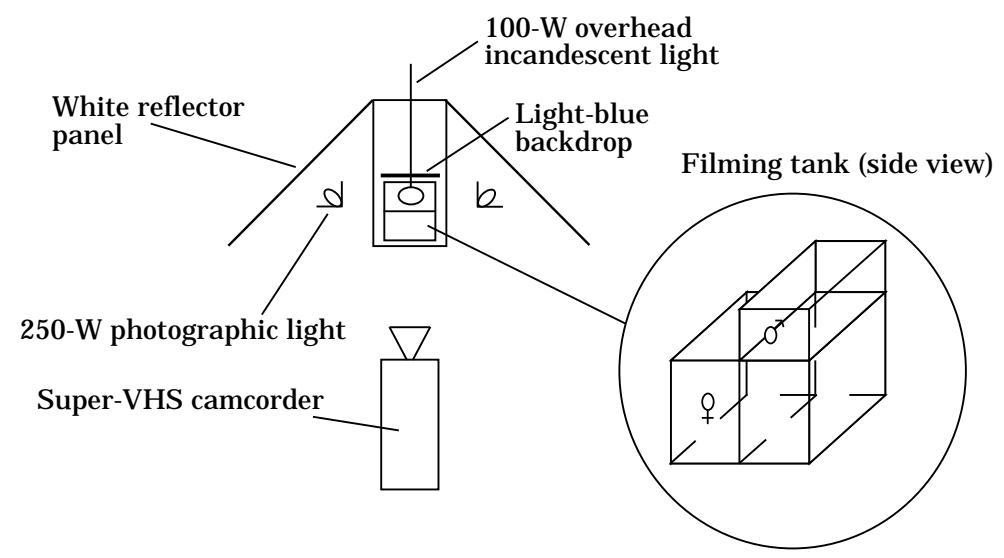

Figure 1. Overhead schematic view of the filming set-up used to obtain stimulus sequences. $M$ ale display behaviour was oriented towards the female, which was confined to a compartment between the male and the camera, and below the male in the camera's field of view.

static morphological attributes already known to play a role in mate choice (Basolo 1990b).

\section{METHODS}

\section{Subjects}

Subjects were 24 female $X$. helleri purchased from commercial suppliers. We tried to obtain females that had coloration close to the wildtype (green). We maintained subjects in the laboratory on a 14:10 h light:dark cycle with fluorescent light provided during daylight hours. They were fed daily with TetraM in and TetraR uby fish flakes, and weekly with frozen tubifex worms. Females were housed communally, in a 148-litre tank, at $26-27^{\circ} \mathrm{C}$. A Il females had been isolated from males for at least 1 month. All subjects were sexually mature and had prior experience with mature males. During the 6-day testing period, we isolated individual females in 1-litre $M$ ason jars within the communal tank, with a small amount of J ava moss provided for shelter.

\section{Preparation of $\mathbf{V}$ ideo Stimuli}

We selected three males to provide stimulus sequences in order to sample natural variation in both morphology and behaviour. Each male was video-recorded while engaging in courtship display. We also video-recorded each of the males while feeding, so that we could assess female responsiveness to a non-courtship behaviour that was matched for overall levels of movement. In addition, we edited footage from the recordings of interactions with females during which the male was not moving. These three 'inactive' stimulus sequences were designed to provide direct comparisons of the effects of variation in male activity levels.

We created two control sequences that allowed us to compare female behaviour while a male image was displayed with that when no male was visible. For comparison with the feeding sequences, we recorded three exemplars of food particles moving within a water-filled tank. Finally, we recorded a sequence of the filming tank containing only water. Because the appearance of this last stimulus did not vary, we used only a single exemplar.

\section{R ecording and editing procedures}

We housed males in a 37-litre tank with four or five other males. Conditions were otherwise identical to those for female subjects. $M$ ales $A$ and $B$ had some orange coloration, and male $C$ was completely wildtype in appearance.

During video-recording, we confined males within a glass holding container, $17.5 \mathrm{~cm}$ wide, which was constructed to match the width of the video monitor used for playback presentations (Fig. 1). This small tank was placed within a 37-litre aquarium containing water at an initial 


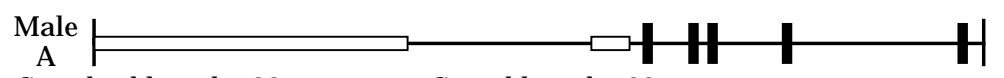

Standard length: $38 \mathrm{~mm} \quad$ Sword length: $39 \mathrm{~mm}$

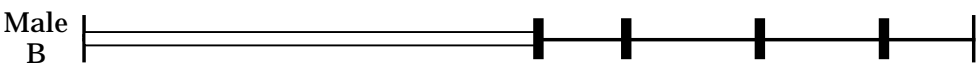

Standard length: $35 \mathrm{~mm} \quad$ Sword length: $32 \mathrm{~mm}$

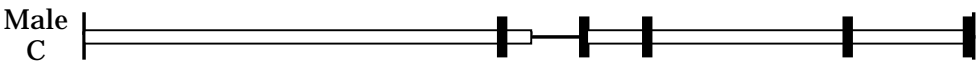

Standard length: $40 \mathrm{~mm} \quad$ Sword length: $40 \mathrm{~mm}$

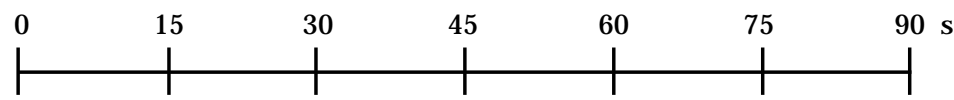

Figure 2. Courtship behaviour and size of males used as test stimuli.

temperature of $27^{\circ} \mathrm{C}$. The holding tank was illuminated by $250-\mathrm{W}\left(3200^{\circ} \mathrm{K}\right)$ photographic lights (Smith-Victor Cal 1) bounced off white card on either side, and by a 100-W incandescent bulb in a metal reflector directly overhead (Fig. 1). These lights typically increased the water temperature $1-2 C^{\circ}$ over the course of a recording session. A sheet of light-blue paper was attached to the back of the tank as a backdrop. We made videorecordings using a Panasonic PVS-350D S-VHS camcorder (resolution >400 lines) with a shutter speed of $1 / 250 \mathrm{~s}$. W e adjusted focal length so that the image obtained on the colour video monitor used for playbacks was precisely life-sized.

We elicited courtship displays by placing the male in the top rear compartment of the holding tank, with a female in the larger compartment below and in front with respect to the camera (Fig. 1). This arrangement prevented the female from obscuring the male, and also ensured that any behaviour pattern directed towards her was also directed towards the camera. The male was allowed to acclimate in an opaque plastic bag within his compartment for approximately $20 \mathrm{~min}$ prior to the start of recording.

Recording sessions lasted until the male had stopped courting the female for more than $5 \mathrm{~min}$. We then selected sequences in which the male performed characteristic display behaviour and edited them together to produce a pattern of continuous courtship activity, while preserving continuity of male movement. M ale courtship displays used in preparing stimuli were those that elicited active interest from females during the recording session itself. These fell into three general categories: (1) a full lateral display, in which the male orients himself perpendicular to the female and swims back and forth with his body slightly shaking (Hemens 1966; Ryan \& Causey 1989; Basolo 1990b); (2) a sigmoidal display, in which the male orients perpendicular to the female with head tilted down and swims back and forth, rapidly curving his body into an S-shape; (3) a backward swim oriented towards the female. Courtship sequences for each male were matched approximately for total activity, but we preserved natural variation in the sequence of display behaviour (Fig. 2). A ll stimuli consisted of a 90 -s sequence looped once for a total of $3 \mathrm{~min}$.

We elicited feeding behaviour by acclimating the males as described above and then adding TetraM in flakes or frozen tubifex worms to the holding tank. R ecording continued until the male had stopped actively feeding. We then edited sequences as described for courtship, making an effort to match the general activity levels of feeding sequences with those of courtship display stimuli.

We prepared the food control sequences by adding food particles to the filming tank and then 
Courtship

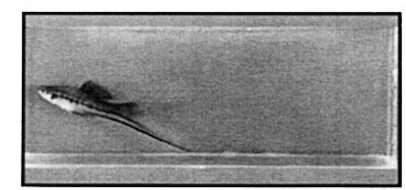

0.5



1.0

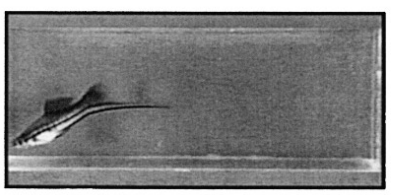

1.5



2.0

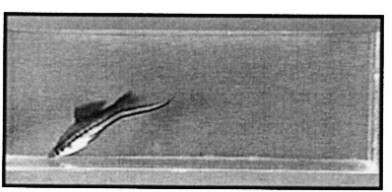

2.5

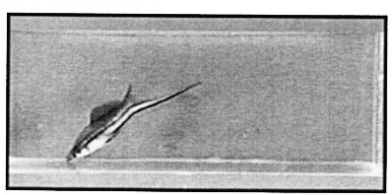

Feeding
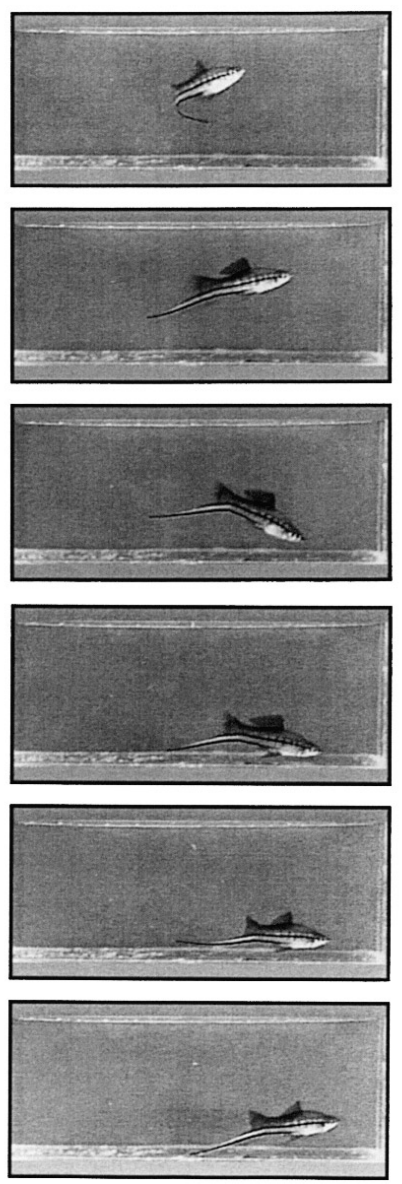

Inactive


Figure 3. R epresentative frames from one exemplar of each type of stimulus sequence depicting a male. A backward swim occurred from 1.0 to $2.5 \mathrm{~s}$ in the courtship sequence.

agitating them with a stream of water, simulating disturbance by an active fish.

We edited raw footage using Panasonic A G-7650 and A G -7750 S-VH S decks. During the editing process, the parts of the image above and below the male were masked by superimposing a computer-generated graphic over the analogue video. This process allowed us to create a 'window' containing the stimulus male and to obscure extraneous elements, such as the female and the water surface. Graphics were created using DeluxePaint IV (Electronic Arts) running on a Commodore A miga 2000 microcomputer with a $D$ igital Creations SuperG en genlock (a device that synchronizes signals from a microcomputer and a video-cassette recorder). Representative frames from each of the three types of stimulus sequence containing a male are shown in Fig. 3 .

\section{Stimulus characteristics}

We examined the completed playback stimuli to quantify differences in the male behaviour depicted. F or each of the nine edited recordings, we sampled every tenth video frame (i.e. 270 frames per sequence). We scored the incidence of three behaviour patterns: (1) 'tail flexes', in which the tail is appreciably curved, (2) 'sigmoid curves', in which the body is curved and (3) backward swims (see above). We also estimated velocity of 
Table I. Characteristics of video stimuli depicting male conspecifics

\begin{tabular}{lcccccc}
\hline Stimulus & Exemplar & $\begin{array}{c}\text { I } \\
\text { Tail } \\
\text { flexes } \\
\text { (frames) }\end{array}$ & $\begin{array}{c}\text { II } \\
\text { Sigmoidal } \\
\text { curves } \\
\text { (frames) }\end{array}$ & $\begin{array}{c}\text { III } \\
\text { Backward } \\
\text { swims } \\
\text { (frames) }\end{array}$ & I and II & $\begin{array}{c}\text { M ean } \\
\text { velocity } \\
\text { (cm/s) }\end{array}$ \\
\hline Courtship & $\mathrm{A}$ & 122 & 62 & 9 & 50 & 2.39 \\
& $\mathrm{~B}$ & 40 & 58 & 17 & 7 & 2.47 \\
F eeding & $\mathrm{C}$ & 57 & 54 & 21 & 8 & 3.56 \\
& $\mathrm{~A}$ & 86 & 42 & 0 & 5 & 2.25 \\
& $\mathrm{~B}$ & 46 & 33 & 0 & 1 & 2.26 \\
& $\mathrm{C}$ & 54 & 22 & 2 & 1 & 2.43 \\
& $\mathrm{~A}$ & 0 & 0 & 0 & 0 & 0.07 \\
& $\mathrm{~B}$ & 0 & 0 & 0 & 0 & 0.02 \\
& $\mathrm{C}$ & 0 & 0 & 0 & 0 & 0.01 \\
\hline
\end{tabular}

movement by comparing the position of the male's eye in successive sample frames.

All six courtship and feeding sequences contained substantial activity, with similar levels of tail flexing and sigmoidal curves (Table I). The most obvious differences between courtship and feeding behaviour were in the incidence of backward swim displays and in the co-occurrence of tail flexes and sigmoid curves; these occurred in all three courtship sequences at qualitatively higher rates than in the feeding sequences (Table I). $\checkmark$ elocity of movement was closely matched in two of the three courtship and feeding exemplars (exemplars $A$ and $B$ ), although the third pair (exemplar C) had a somewhat higher rate of movement in the courtship sequence than in the feeding sequence. Overall, the mean velocity of movement in the feeding sequences was $82.2 \%$ of that in the courtship sequences. Courtship movements appeared rather more abrupt and discontinuous than those characteristic of feeding behaviour.

The inactive sequences were clearly distinct from both feeding and courtship sequences. N one of the three behaviour patterns described above occurred in these stimuli (T able I). In addition, the level of movement was only about $1 \%$ of that apparent in the other two types of stimulus sequences.

\section{P layback P resentations}

Each female was randomly assigned one of the three stimulus sets. She was then shown all five stimulus types in a random order, receiving each playback on a different day. To control for diel differences in receptivity, females were shown all stimuli at the same time of day $( \pm 45 \mathrm{~min})$. Females 1-12 received stimuli in the morning (from $30 \mathrm{~min}$ after the beginning of the light cycle to $5 \mathrm{~h}$ thereafter), and females 13-24 received stimuli in the afternoon (from 8 to $13 \mathrm{~h}$ into the light cycle). A the beginning of each test session, we removed the females to be used and placed them in a 37-litre tank. The testing tank was then filled with water from their home aquarium to minimize temperature differences in acclimation. All females were fed to satiation 40 min prior to testing.

We tested females in a 37-litre tank $(50.8 \times 26.0 \times 31.75 \mathrm{~cm})$ with a Panasonic BT-S901Y S-VHS colour monitor (screen size $22.9 \mathrm{~cm}$ measured diagonally) abutting one end. We played all presentation sequences on a Panasonic A G -7650 S-VH S video-cassette player. The experimenter was concealed behind a large opaque barrier. We recorded tests using a videocamera that was positioned at right angles to the testing tank so that the female's response was visible to the experimenter, but the stimulus image was not.

Each female was acclimated in the testing tank for $20 \mathrm{~min}$, after which she was shown a 10-min empty-tank sequence, followed by the 3-min stimulus and then a further 3 min of the emptytank image. We video-recorded females for the duration of the stimulus, and for 3-min prestimulus and post-stimulus periods. At the end of the post-stimulus period, the female was returned to her home tank. 


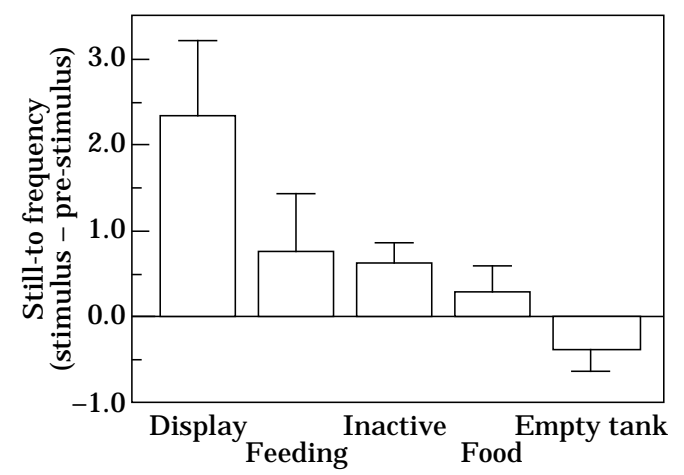

Figure 4. Still-to frequency difference scores $(\bar{X}+\mathrm{sE})$ for each type of video stimulus.

\section{D ata A nalysis}

We operationally defined females as showing active interest when they turned to face the monitor screen mid-water (not resting at the surface or on the bottom) and stopped moving. We recorded the frequency of this behaviour pattern (referred to as 'Still-to'). A number of previous studies (Hemens 1966; Ryan \& Causey 1989; Basolo 1990b) have used this type of behaviour as an indicator of female responsiveness to courting male conspecifics. In addition, our pilot work with video stimuli suggested that Still-to was the most reliable indicator of active interest.

We scored the frequency of Still-to responses from test session video-recordings using eventrecorder software running on an Apple $\mathrm{II}+$ microcomputer (timing resolution $100 \mathrm{~ms}$ ). All behaviour was judged 'blind', with the observer unaware of the type of video stimulus to which the female was responding. For statistical analysis, we pooled the responses elicited by all three exemplars of each type.

We first assessed the absolute effectiveness of each stimulus type by conducting a Friedman non-parametric ANOVA on the data from prestimulus, stimulus and post-stimulus periods. These analyses established whether female behaviour patterns had changed significantly over the course of the test session.

F emales varied considerably in the frequency of Still-to responses during the pre-stimulus period. We therefore conducted analyses of behaviour during the stimulus and post-stimulus periods using difference scores, subtracting the prestimulus Still-to score for each presentation from the stimulus and post-stimulus scores. We then compared the responses elicited by all five stimulus types using F riedman non-parametric ANOVAs performed separately for the pre-stimulus and stimulus periods. Comparisons yielding significant main effects were subsequently analysed with Wilcoxon signed-rank tests.

We predicted that courtship behaviour would elicit a greater response than any other stimulus and that video sequences including males would be more attractive than the food and empty tank controls. Statistical comparisons based upon these a priori predictions were one-tailed; all other comparisons were two-tailed. We excluded three females from the analysis; one died within 2 days of the last presentation, one bore fry during a test trial, and one was inadvertently shown a stimulus out of sequence.

\section{RESULT S}

There were significant differences in the frequency of female Still-to responses across the pre stimulus, stimulus and post-stimulus periods for the courtship stimulus $\left(\chi^{2}=12.75, \mathrm{df}=2, \mathrm{P}<0.005\right)$ and for the feeding $\left(\chi^{2}=6.41, d f=2, P<0.05\right)$ and inactive stimuli $\left(\chi^{2}=6.0, d f=2, P<0.05\right)$, but not for the food $\left(\chi^{2}=0.89, d f=2, P=0.64\right)$ or empty tank controls $\left(\chi^{2}=1.72, \mathrm{df}=2, \mathrm{P}=0.42\right)$. F emale behaviour patterns were thus affected by all three stimuli that presented images of conspecific males, but not by either of the control stimuli.

A nalyses of female behaviour patterns during stimulus presentations demonstrated significant differences in the magnitude of the responses evoked by the five types of video sequences ( $F$ ig. 4; $\chi^{2}=11.17, \mathrm{df}=4, \mathrm{P}<0.05$ ). F emale behaviour patterns during the post-stimulus periods did not differ reliably as a function of preceding stimulus type $\left(\chi^{2}=8.51, d f=4, P=0.07\right)$, although the relatively small sample size and high degree of variability between subjects may contribute to the failure of this difference to reach statistical significance.

Pair-wise comparisons revealed that courtship displays elicited a significantly higher frequency of Still-to responses than any other stimulus (Fig. 4; smallest $\mathrm{Z}=-1.69, \mathrm{~N}=21, \mathrm{P}<0.05)$. Inactive males elicited significantly higher rates of responding than the empty tank control $(z=2.67, N=21$, $P<0.004)$. There was no significant difference in 
the responses evoked by the feeding and inactive sequences $(z=-0.38, N=21, P=0.70)$, nor was there a significant difference between the responses to the feeding stimulus and those to the food control $(z=-0.67, N=21, P=0.50)$. F emales did not respond differently to the food and empty tank controls ( $z=-1.29, \mathrm{~N}=21, \mathrm{P}=0.20)$. Video sequences of courting males thus elicited significantly more Still-to responses than other stimuli, including the feeding sequences which presented females with comparable levels of male activity.

\section{DISCUSSION}

The results of this playback experiment demonstrate that female swordtails respond socially to video sequences of conspecific males. Female behaviour patterns changed significantly when an image of a male swordtail was visible, but not with control images. There is also evidence that female responsiveness was affected by the type of male behaviour presented, even though there was no accompanying variation in morphology. Courtship displays elicited significantly more responses than sequences depicting either a male engaged in other activity or an inactive male.

The significant difference between the overall effects of courtship and feeding sequences may be a consequence of the slightly higher velocity of movement in one of the three courtship stimuli (Table I). We believe this to be unlikely, however, because we were unable to detect an effect of activity level in other comparisons. F or example, female behaviour patterns were unaffected by qualitatively larger differences in movement velocity between the feeding and inactive sequences (Table I). Similarly, the feeding male stimulus did not elicit significantly more Still-to responses than the control sequence showing only moving food particles. These results would not have been expected if female responsiveness was principally dependent upon the level of gross activity in the video image.

Our results suggest that female preference in $X$. helleri is influenced by dynamic traits such as the incidence of courtship behaviour. This finding is consistent with those of previous studies implicating male behaviour patterns in female mate choice decisions. F or example, F arr (1980) found that the preference of female guppies for brighter males (K odric-Brown 1985) could be reversed by variation in courtship behaviour; females preferred dull-coloured males with high display rates to brightly coloured males with low display rates.

The results of the present study complement Basolo's (1990b) demonstration that female swordtails attend to a morphological character, sword length. Static traits of this type provide females with information about a male's condition integrated over a relatively long period (e.g. $\mathrm{H}$ amilton \& Zuk 1982), while dynamic traits allow an assessment of current male condition. We suggest that the sword and courtship display may act synergistically to determine female preference.

It is likely that courtship motor patterns have coevolved with elements of male morphology. In many species, conspicuous male ornaments are coupled with stereotyped movements that present them to best advantage (e.g. peacocks, Pavo cristatus: Tomlinson \& O’D onald 1989; jungle fowl: Zuk et al. 1992). Courtship behaviour in $X$. helleri may similarly interact with the sword to present females with a more conspicuous visual stimulus. The backward swim is a particularly likely candidate for such a role, because this motor pattern sweeps the sword back and forth across the female's visual field. Intriguingly, the teleostean optic tectum, which is involved in the control of orienting and pursuit responses (Springer et al. 1977; Y ager et al. 1977; V anegas 1983), contains neural structures that show pronounced directional biases in their sensitivity to moving images (Guthrie 1990). Characteristic courtship displays may thus have evolved to trigger the higher levels of sensory processing that determine behavioural responses.

Video playback experiments provide a particularly powerful range of techniques for mapping the interactive effects of static and dynamic traits on female responses. We have used video stimuli to show that females prefer a male performing a specific set of motor patterns to a similarly active male engaged in other behaviour patterns. Further experiments will involve altering the relative frequency of selected components of courtship; for example, increasing or decreasing the number of backward swims. V ideo sequences could also be used in combination with computer animation techniques to compare the effects of manipulating male morphology (e.g. removing the sword) with those of altering the parameters of dynamic patterns (e.g. increasing the visual angle subtended by a backward swim). A nalyses of this type would 
allow us to model the way that females 'weight' various male attributes in mate choice decisions, in much the same way as has been done for the acoustic characters mediating bird song recognition in an agonistic context (e.g. N elson 1988).

It might also be revealing to search for a predisposition to respond to courtship behaviour in species where such behaviour is absent. For example, $X$.pygmaeus females prefer male $X$. nigrensis, which are larger, have swords, and perform courtship (Ryan \& Wagner 1987). Digitized video stimuli can be used to assess female response to each of these components independently. These and similar experiments would provide a means of identifying the perceptual cues that influence female choice and the evolution of male traits.

\section{A C K N O W LE DG MENTS}

We are especially grateful to Peter M arler for providing us with laboratory resources and access to equipment. A n N SF R esearch Training G rant awarded to the $U$ niversity of California at $D$ avis, provided the video-cassette recorders and monitor used in the playback experiment. G.G.R. is indebted to the same RTG for an undergraduate summer fellowship which provided support for the initial stage of the research. A portion of this work was done in partial fulfilment of G.G.R.'s undergraduate honours thesis at $\mathrm{H}$ arvard U niversity and was supported by the Ford Foundation for U ndergraduate R esearch. We thank (in chronological order) Joseph M acedonia, A lexandra Basolo, John Endler, K arel Liem, Evan Balaban, Edward O. Wilson, M arc D antzker, A. Stanley $\mathrm{R}$ and, $\mathrm{M}$ ichael J. R yan, Ingo Schlupp, C atherine $M$ arler, Laurie Dries, Paige Warren, Becki Thompson, H. Carl Gerhardt, David Clark and one anonymous referee for comments on drafts of the manuscript.

\section{REFERENCES}

A ndersson, M. 1982. F emale choice selects for extreme tail length in a widowbird. Nature, L ond., 299, 818820.

Baker, M . C., Bjerke, T. K ., L ampe, H .\& Espmark, Y. 1986. Sexual response of female great tits to variation in the size of males' repertoires. Am. Nat., 128, 491-498.
Basolo, A. L. 1990a. F emale preference predates the evolution of the sword in swordtail fish. Science, 250, 808-810.

Basolo, A. L. 1990b. Female preference for male sword length in the green swordtail (Pisces: Poeciliidae). A nim. B ehav., 40, 332-338.

Bischoff, J. A., Gould, J. L. \& R ubenstein, D. I. 1985. Tail size and female choice in the guppy (Poecilia reticulata). Behav. E col. Sociobiol., 17, 253-255.

Burley, N. 1981. M ate choice by multiple criteria in a monogamous species. A m. N at., 117, 515-528.

Clark, D. L. \& U etz, G. W. 1990. Video image recognition by the jumping spider, $M$ aevia inclemens (A raneae: Salticidae). A nim. B ehav., 40, 884-890.

Clark, D. L. \& U etz, G. W . 1992. M orph-independent mate selection in a dimorphic jumping spider: demonstration of movement bias in female choice using video-controlled courtship behaviour. A nim. Behav., 43, 247-254.

Clark, E., A ronson, L. R . \& Gordon, M . 1954. M ating behavior patterns in two sympatric species of xiphophorin fishes: their inheritance and significance in sexual isolation. Bull. A m. M us. nat. $\mathrm{H}$ ist., 103, 135226.

Clayton, D. H. 1990. M ate choice in experimentally parasitized rock doves: lousy males lose. Am. Z ool., 30, 251-262.

Collins, S. A. 1994. M ale displays: cause or effect of female preference. A nim. B ehav., 48, 371-375.

Darwin, C. 1871. The Descent of $M$ an, and Selection in Relation to Sex. London: J ohn M urray.

Endler, J. A . 1980. N atural selection on color patterns in P oecilia reticulata. Evolution, 31, 76-91.

Endler, J. A. 1992. Signals, signal conditions, and the direction of evolution. Am. Nat., 139, 1-27.

Evans, C. S. \& M arler, P. 1991. On the use of video images as social stimuli in birds: audience effects on alarm calling. A nim. B ehav., 41, 17-26.

Evans, C. S. \& M arler, P. 1992. F emale appearance as a factor in the responsiveness of male chickens during anti-predator behaviour and courtship. A nim. B ehav., 43, 137-143.

Evans, C. S., Evans, L. \& M arler, P. 1993a. On the meaning of alarm calls: functional reference in an avian vocal system. A nim. B ehav., 46, 23-38.

Evans, C. S., M acedonia, J. M.\& M arler, P. 1993b. Effects of apparent size and speed on the response of chickens, Gallus gallus, to computer-generated simulations of aerial predators. A nim. Behav., 46, 1-11.

Farr, J. A. 1980. Social behaviour patterns as determinants of reproductive success in the guppy, P oecilia reticulata Peters (Pisces: Poeciliidae). B ehaviour, 74, 38-91.

Franck, D. 1964. Vergleichende Verhaltensstudien an lebendgebaren Zahnkarpfen der Gattung Xiphophorus. Z ool. J ahrb. P hysiol. Bd, 71, 117-170.

Gerhardt, C. 1988. A coustic properties used in call recognition by frogs and toads. In: The Evolution of the A mphibian A uditory System ( $E d$. by B. F ritzsch et al.), pp. 455-483. N ew Y ork: John Wiley.

Gerhardt, H. C. 1991. F emale mate choice in treefrogs: static and dynamic acoustic criteria. A nim. Behav., 42, 615-635. 
G uthrie, S. 1990. The physiology of the teleostean optic tectum. In: The Visual System of Fish (Ed. by R. Douglas \& M. Djamgoz), pp. 279-344. London: Chapman \& Hall.

H amilton, W. D. \& Zuk, M . 1982. H eritable true fitness and bright birds: a role for parasites? Science, 218, 384-387.

Hemens, J. 1966. The ethological significance of the sword-tail in Xiphophorus hellerii [sic] (H aeckel). Behaviour, 27, 290-315.

H ill, G. E. 1990. F emale house finches prefer colourful males: sexual selection for a condition-dependent trait. A nim. Behav., 40, 563-572.

Houde, A. E. 1987. M ate choice based upon naturally occurring color-pattern variation in a guppy population. Evolution, 41, 1-10.

Kodric-Brown, A. 1985. F emale preference and sexual selection for male coloration in the guppy (Poecilia reticulata). Behav. E col. Sociobiol., 17, 199-205.

M acedonia, J. M., Evans, C. S. \& Losos, J. B. 1994. $M$ ale A nolis lizards discriminate video-recorded conspecific and heterospecific displays. A nim. B ehav., 47, 1220-1223.

M cG regor, P. K. (Ed.) 1992. Playback and Studies of A nimal Communication. $\mathrm{N}$ ew $\mathrm{Y}$ ork: Plenum Press.

N elson, D. A. 1988. Feature weighting in species song recognition by the field sparrow (Spizella pusilla). B ehaviour, 106, 158-182.

Proctor, H. C. 1991. Courtship in the water mite $\mathrm{N}$ eumania papillator: males capitalize on female adaptations for predation. A nim. B ehav., 42, 589-598.

Ryan, M. J. 1990. Sexual selection, sensory systems, and sensory exploitation. Oxf. Surv. evol. Biol., 7, 157-195.

R yan, M. J. 1991. Sexual selection and communication in frogs. Trends E col. E vol., 6, 351-354.
Ryan, M.J . \& Causey, B. A . 1989. 'A Iternative' mating behaviour in the swordtails $X$ iphophorus nigrensis and $X$ iphophorus pygmaeus (Pisces: Poeciliidae). Behav. E col. Sociobiol., 24, 341-348.

R yan, M. J. \& Wagner, W. E., J r. 1987. A symmetries in mating preferences between species: female swordtails prefer heterospecific males. Science, 236, 595597.

Ryan, M. J., Hews, D. K. \& Wagner, W. E., J r. 1990. Sexual selection on alleles that determine body size in the swordtail Xiphophorus nigrensis. Behav. Ecol. Sociobiol., 26, 231-237.

Schlosberg, H. M., D uncan, M. C. \& Daitch, B. H. 1949. $M$ ating behavior of two live-bearing fish, $X$ iphophorus helleri and Platypoecilius maculatus. Physiol. Zool., 22, 148-161.

Springer, A . D ., E aster, S. S., J r \& A granoff, B. W . 1977. The role of the optic tectum in various visually mediated behaviors of the goldfish. Brain Res., 128, 393-404.

Tomlinson, I. \& O'D onald, P. 1989. The co-evolution of multiple female mating preferences and preferred male characters: the gene-for-gene hypothesis of sexual selection. J . theor. Biol., 139, 219-238.

Vanegas, H. 1983. Organization and physiology of the teleostean optic tectum. In: Fish N eurobiology ( $\mathrm{Ed}$. by R. E. Davis \& R. G. Northcutt), pp. 43-90. Ann A rbor: U niversity of $M$ ichigan Press.

Y ager, D., Sharma, S. C. \& Grover, B. G. 1977. Visual function in goldfish with unilateral and bilateral tectal ablation. Brain Res., 137, 267-276.

Zuk, M., Ligon, J. D. \& Thornhill, R. 1992. Effects of experimental manipulation of male secondary sex characters on female mate preference in red jungle fowl. A nim. Behav., 44, 999-1006. 